2018-09

\title{
Electrophysiological study of
}

action-affordance priming between

object names.

\section{Feven-Parsons, IM}

http://hdl.handle.net/10026.1/11799

\author{
10.1016/j.bandl.2018.06.002 \\ Brain and Language \\ Elsevier
}

All content in PEARL is protected by copyright law. Author manuscripts are made available in accordance with publisher policies. Please cite only the published version using the details provided on the item record or document. In the absence of an open licence (e.g. Creative Commons), permissions for further reuse of content should be sought from the publisher or author. 


\title{
Electrophysiological study of action-affordance priming between object
} names

\author{
Isabel M. Feven-Parsons ${ }^{*}$, Jeremy Goslin \\ School of Psychology, University of Plymouth, Plymouth, Devon PL4 8AA, UK
}

\section{A B S T R A C T}

If our central representation of an object is defined through embodied experience, we might expect access to action affordances to be privileged over more abstract concepts. We used event-related potentials to examine the relative time course of access to affordances. Written object names were primed with the name of an object sharing the same affordance as the target (e.g. precision-grip: "grape" primed by "tweezers") or the same taxonomic category (e.g. fruit: "grape" primed by "apple"). N200 latencies, related to go/nogo semantic cate- gory decisions on target words, revealed no difference in facilitation provided by affordance and semantic priming. However, separate analyses of ERPs for go and nogo trials showed that semantic priming led to earlier activation during go trials (around $430 \mathrm{~ms}$ ), and affordance priming led to earlier activation during nogo trials (around $180 \mathrm{~ms}$ ). While affordances appear to be peripheral to the conceptual representation of objects, they do lead to direct motor preparation.

\section{Introduction}

The majority of the embodied cognition literature has focused on the sensorimotor processes involved in processing action words and sentences. However, it is also thought that the name of an object will also recruit the sensorimotor brain activity associated with the referent's form and function, such as its action affordances (Bub \& Masson, 2012). Affordances are the behavioural possibilities provided by the environment and are detected automatically by the visual system, regardless of the organism's intention to act (Garbarini \& Adenzato, 2004; Gibson, 1979). For example a mug affords being grasped with the hand (Withagen, de Poel, Araújo, \& Pepping, 2012). The Indexical Hypothesis proposes that nouns are indexed to mental representations (such as mental pictures) of the objects they refer to (Glenberg \& Robertson, 1999). Subsequently, when a noun is processed the affordances of the referent object are made available. According to the Indexical Hypothesis accessing the affordances of referent objects is crucial for noun comprehension (Glenberg \& Gallese, 2012; Glenberg \& Robertson, 1999). A number of behavioural studies support the idea that affordances are retrieved during object name processing (Barbieri, Buonocore, Bernardis, Dalla Volta, \& Gentilucci, 2007; Bub \& Masson, 2012; Bub, Masson, \& Cree, 2008; Gentilucci \& Gangitano, 1998; Glover \& Dixon, 2002; Glover, Rosenbaum, Graham, \& Dixon, 2004; Marino, Gough, Gallese, Riggio, \& Buccino, 2013; Myung, Blumstein, \& Sedivy, 2006; Tucker \& Ellis, 2004). Participants are quicker to make categorical judgements when responding with a hand-grip that would be used to interact with the referent object (Tucker \& Ellis, 2004; experiment 3). Furthermore, reading the name of a manipulable object activates areas of the premotor cortex which are also involved in action word processing (Grabowski, Damasio, \& Damasio, 1998).

While the interaction between motor and language processing is well evidenced, the utility of this relationship is an area of considerable controversy. There seems to be little doubt that linguistic representations of actions and affordances can generate motor activity, but it is unclear what, if any, role this activity plays in language comprehension (Chatterjee, 2010; Dove, 2009, 2011; Mahon \& Caramazza, 2008). Mahon and Caramazza (2008) argue that findings purported to support embodied cognition could just as easily be explained by a disembodied account. They propose that an initial retrieval of abstract concepts is followed by the spreading of activation to sensory and motor areas, with these activations reflecting a later epiphenomenal process, such as mental imagery. Language and situated simulation (LASS) theory proposes that two systems are involved in language comprehension (Barsalou, Santos, Simmons \& Wilson, 2008). The Linguistic system identifies the word form and the simulation system activates perceptual and experiential information associated with the object, such as its affordances. The linguistic system is sufficient for resolving tasks that only require shallow processing, involving statistical relations between words in a semantic network (words associated through frequent cooccurrence). For deep and meaningful processing, simulations are 
necessary and these are activated automatically, within $200 \mathrm{~ms}$ after word onset. It is important to understand when, and by implication what stage of processing, language perception makes use of embodied representations. If they are fundamental to the conceptual representations of objects it might be expected that they would be available in advance of more abstract information. This kind of temporal information can be difficult to ascertain with behavioural experiments, but is particularly well suited to the ERP technique. Amsel, Urbach and Kutas (2013) used this technique to determine the temporal order of access to abstract and motor related semantic information when presented with the names of objects. Using a go/nogo task they compared the temporal onset of the N200 component when participants were asked to make a judgment on whether objects were graspable or non-graspable, or whether they were living or non-living. The N200 is a negative going component resulting from the subtraction of go from nogo trials, and is thought to provide an indication about when sufficient information has become available to allow a participant to make or withhold their response (Augustin, Defranceschi, Fuchs, Carbon, \& Hutzler, 2011). Amsel et al. (2013) found that the onset of the N200 related to a living/ non-living judgment was at around $160 \mathrm{~ms}$ after stimulus presentation, compared to $300 \mathrm{~ms}$ for the graspable/non-graspable judgment. The relatively late access to grasp-related affordances prompted the authors to conclude that they did not play a crucial role in the conceptual representation of objects.

Although the results provided by Amsel et al. (2013) seem relatively clear, they are based upon the assumption that the participant has direct access to the information relevant to this explicit decision. However, affordances are generally considered to be processed automatically as a component of object representation that provides implicit facilitation of a wide range of responses (Barbieri et al., 2007; Glover et al., 2004; Marino et al., 2013; Myung et al., 2006; Pulvermüller, Shtyrov, \& Ilmoniemi, 2005; Tucker \& Ellis, 2004). For example, Glover et al. (2004) found that when participants went to grasp a wooden block, the aperture of their grip was larger when they read a word referring to a large object than a small object. Another study found that when participants heard the names of objects they spent longer looking toward pictures of objects that shared similar manipulation features with the named object than those that did not, with affordance-related looking occurring as early as $300 \mathrm{~ms}$ (Myung et al., 2006; experiment 2). As it has been established that affordance modulates behaviour without the awareness of the participant, it is possible that the earliest access to this property may not be revealed through explicit questioning.

In our study we wanted to capture the implicit effects of affordance in an ERP study similar to that of Amsel et al. (2013). However, instead of comparing the N200 related to different explicit judgment decisions, i.e. those based on semantic and affordance information, we examined how the N200 related to the same semantic decision would be modulated by priming. Semantic priming effects are well established in the literature (Lucas, 2000), with priming found to improve both the accuracy and reaction times in lexical decision tasks (Meyer \& Schvaneveldt, 1971). This facilitation is also found when the prime is masked (e.g. Forster \& Davis, 1984), that is when presented for a very short duration (50-60 ms), usually sandwiched between two visually obscuring forward and backward masks. This is designed to allow the investigation of the prime-target relationship without the awareness of the participant, so preventing the use of explicit response strategies. Studies using the ERP technique have shown that semantically related prime-target pairs elicit a smaller N400 than semantically unrelated prime-target pairs (Deacon, Hewitt, Yang, \& Nagata, 2000; Kutas \& Federmeier, 2011). This is thought to reflect the greater ease in which the target is integrated into the semantic context provided by the prime (Borovsky, Elman, \& Kutas, 2012). In most studies, the semantic relatedness between prime and target is determined by semantic category norms, such as those of Battig and Montague (1969), which largely shared a taxonomic relationship (e.g. steel and iron being "types of metal"). There are few studies that have examined the relationship between objects formed by a shared affordance. In one such study by Myung et al. (2006), auditory prime and target words either shared similar manipulation features (e.g. piano and typewriter) or not (e.g. piano and blanket). This study showed that shared affordances facilitated reaction times, but did not provide any direct comparison with the facilitation provided by taxonomic semantic priming.

Here we have used the masked priming paradigm to compare the relative differences in priming between written prime-target word pairs that are related either through taxonomy (e.g. "grape" and "banana" are both fruit) or affordance (e.g. "hammer" and carrot" are both manipulated using a power-grip). Both of these related priming conditions were also compared to a baseline condition, where the prime did not share the same taxonomy or affordance with the target (e.g. "mushroom" and "drill"). These word pairs were used in a go/nogo task to evoke an N200 component related to a speeded natural/manmade decision on the target words. An estimate of the temporal onset of affordance and general semantic information was provided through a comparison of the N200 between the three priming conditions. If the sensorimotor activity associated with affordances is fundamental to object representation, then we would hypothesise that the facilitation provided by the priming of affordances should occur earlier than that of semantic priming. Conversely, if affordances are produced as part of a post-lexical mental simulation of object use or accessed via an amodal process of spreading activation we would expect that the temporal onset of this information should occur after semantic processing.

\section{Method}

\subsection{Participants}

Sixty native monolingual English speakers gave informed, written consent to participate in the experiment and were paid $£ 12$ for their participation. The Data from 9 participants was discarded due to excessive EEG and electrooculography (EOG; eye movements) artefacts (less than $66 \%$ of recorded trials available for analysis). The remaining 51 participants ( 32 female) were aged between 18 and 32 (mean age = 21.76). All participants were right-handed (as assessed with the Edinburgh Handedness Inventory; Oldfield, 1971), reported having normal or corrected-to-normal vision and had no history of neurological impairment.

\subsection{Stimuli}

The critical stimuli consisted of 32 different concrete nouns (taken from the CELEX database; Baayan, Piepenbrock, \& Gulikers, 1995) referring to manually manipulable objects. Sixteen of the nouns were used as prime words (3-10 letters in length) and 16 were used as the target words (3-6 letters in length). Half of the prime words referred to natural objects and half referred to manmade objects. Within each of those categories, half were the names of objects affording a power-grip (e.g. "hammer" or "carrot") and half were names of objects affording a precision-grip ("scalpel" or "grape"). The target words were a different set of 16 nouns that were also equally divided between these four categories (manmade power-grip; manmade precision-grip; natural powergrip; natural precision-grip).

Each prime word was paired with each target word so that there were 256 prime-target pairs. There were three conditions: semantic (when the prime and target referred to objects that were taxonomically related but did not afford the same grip e.g. 'strawberry-banana' or 'potato-pea'); affordance (when the prime and target referred to objects that afforded the same hand grip but were not taxonomically related e.g. 'tweezers-lentil' or 'orange-axe'); or neutral (when the prime and target referred to objects that were neither semantically related nor afforded the same grip e.g. 'fig-hammer' or 'scalpel-apple'; see Appendix for a full list of the stimuli). There were 64 different prime- 

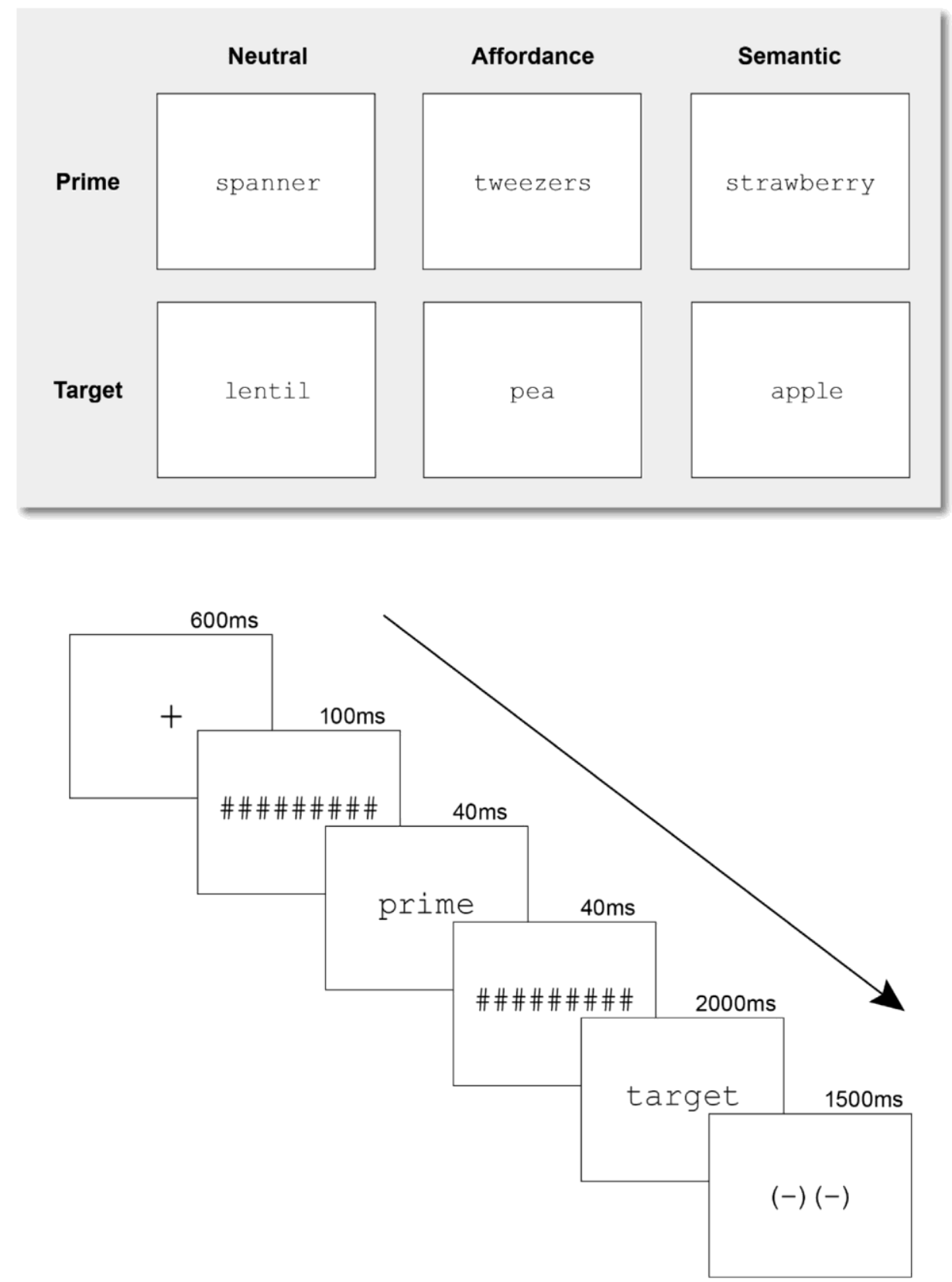

Fig. 1. Example of stimuli presented in the experiment (a) and an illustration of the experimental sequence (b).

target pairs in each condition. The remaining prime-target pairs were used as filler stimuli.

\subsection{Procedure}

Participants were presented the stimuli on a CRT monitor $(30.5 \mathrm{~cm}$ height by $40.5 \mathrm{~cm}$ width; $100 \mathrm{~Hz}$ refresh rate) positioned at eye level one metre from the participant in a quiet dimly-lit booth. Stimuli were presented using E-Prime 2.0 (Schneider \& Zuccoloto, 2007), with responses collected using an E-Prime button box.

The sequence of each trial was as follows. At the beginning of each trial, a fixation point, " + ", appeared at the centre of the screen for 600-800 ms. The fixation point was followed by a forward mask (\#\#\#\#\#\#\#\#\#) for $100 \mathrm{~ms}$. This was followed by a prime word presented for $40 \mathrm{~ms}$ and then a backward mask (\#\#\#\#\#\#\#\#\#) for
$40 \mathrm{~ms}$. The target was then presented for up to $2000 \mathrm{~ms}$ or until the participant responded. At the end of each trial a blink symbol, "(-) $(-)$ ", was displayed for $1500 \mathrm{~ms}$, giving the participant the opportunity to blink if necessary (see Fig. 1 for example stimuli and an illustration of the experimental sequence). The participants were asked to avoid making eye movements or blinks until the blink symbol was displayed in order to reduce contamination of the EEG data. All text was displayed in the Courier New typeface in black on a white background. Participants responded to the target words using the index finger of their left hand. This was to make the response as unrelated to the object affordance as possible because the participants were all right-handed and would therefore usually pick up the objects with their right hand. A go/nogo paradigm in two between-participants response conditions was adopted whereby half of the participants were required to respond only to natural stimuli and to withhold responses to manmade stimuli. 
The other half were required only to respond to manmade stimuli and to withhold a response when they saw natural stimuli. Participants were instructed to indicate as quickly and accurately as possible whether the word referred to the semantic category to which they had been assigned (i.e. natural or manmade).

A sequence of 18 practice trials (using separate stimuli that were not used in the main experiment) was completed by each participant and could be repeated if necessary. After this each of the 256 prime-target word pairs were presented three times in three seamless blocks, resulting in 576 critical trials and 192 filler trials. Trials with slow (> $1200 \mathrm{~ms}$ ) or incorrect responses were excluded from further analysis ( $1 \%$ of trials). Trials were presented continuously with participants being provided with a 'rest period' after every 90 trials.

\subsection{EEG recording}

BrainVision Recorder (Version 1.10, Brain Products GmbH) was used to collect the scalp voltages from $61 \mathrm{Ag} / \mathrm{AgCl}$ active electrodes (actiCAP, Brain Products, Gilching, Germany). The sensors were ar-ranged in the International 10-20 configuration and secured in place on the participant's scalp by an elastic cap. An additional two sensors were positioned below and adjacent to the participant's right eye to monitor eye movements. Segments of EEG data containing eye movement or blink artefacts were not included in later analyses. All scalp electrode impedance measurements were kept below $20 \mathrm{k} \Omega$. The EEG signals were amplified by a BrainAmp MR Plus amplifier (Brain Products).

\subsection{EEG analyses}

Vision Analyser (Version 2.0, Brain Products GmbH) was used to process the data. EEG was sampled at a rate of $250 \mathrm{~Hz}$ and filtered offline with a band-pass filter of $0.1-40 \mathrm{~Hz}$ (with a roll-off slope of 12 $\mathrm{dB} /$ oct) and subjected to a $50 \mathrm{~Hz}$ notch filter. The EEG recordings were segmented into $1000 \mathrm{~ms}$ epochs, spanning from $200 \mathrm{~ms}$ before the onset of the target word until $800 \mathrm{~ms}$ afterwards. Separate ERPs were generated for the same set of target word stimuli presented in three different priming conditions (semantic, affordance and neutral priming). Baseline correction was performed using the average EEG activity between $-200 \mathrm{~ms}$ and $0 \mathrm{~ms}$. The electrodes were referenced to the left mastoid electrode and then re-referenced offline to the average of the left and right mastoid data. The central anterior-frontal electrode (AFz) was used as the ground. Segments containing artefacts were re-jected from analyses and participants with less than two thirds of their segments intact after artefact removal were excluded from the analyses. Inaccurate responses were discarded, as were trials with reaction times 2.5 standard deviations above or below the mean, or outside the 2001200 ms time window.

To calculate the N200, the go data was subtracted from the nogo data for each condition (Amsel et al., 2013; Schmitt, Münte, \& Kutas, 2000). Comparisons between conditions were conducted across all electrodes and post zero-point sample points using pairwise analyses based upon the cluster randomisation technique of Maris and Oostenveld (2007), to avoid multiple comparisons. In this technique, two-sided $t$-tests were carried out comparing each electrode-time sample pair between two of the tested conditions (e.g. affordance and semantic priming). Those samples with a $t$-value above significance threshold ( $\mathrm{p}$ $<.05$ ) were clustered together in terms of temporal and spatial adjacency. Only clusters of eight or more samples were con-sidered for analysis. For each of the remaining clusters a summed $t$-value was calculated as a total of all individual $t$-values from all of the individual comparisons. Analysis thereafter was based on these clusters rather than the individual data points. In the second step of this pro-cedure the interval occupied by the cluster with the largest cluster-level $t$-value was selected. Each of the original paired sample $t$-tests that were used to generate this cluster were repeated, but with the data items of each pair randomly assigned between the two conditions. This was performed 1000 times to generate a Monte Carlo distribution of summed $t$-values corresponding to the null hypothesis. The final Monte Carlo $p$-value was calculated as the proportion of the 1000 summed $t$ values in the random distribution that exceeded the observed clusterlevel $t$ statistic.

\section{Results}

\subsection{Behavioural results}

Accuracy data. The average proportion of correct responses across all conditions was found to be $99.44 \%$. An ANOVA was conducted comparing accuracy between the 3 conditions (semantic, affordance, neutral), 2 response types (go, nogo) and 2 categories (manmade, natural). There were no significant effects, $p \mathrm{~s}>.1$.

Reaction Times. An ANOVA was conducted comparing the 3 conditions (semantic, affordance, neutral) and 2 categories (manmade, natural). A significant main effect of condition was found, $F(3$, $147)=9.89, p<.0001$, with participants responding significantly quicker on the semantic primed trials $(M=572.38 \mathrm{~ms}, S D=90.78)$ compared to the affordance trials $(M=587.97 \mathrm{~ms}, S D=90.78 ; F(1$ ,49) $=11.39, p<.01)$, and the neutral trials $(M=587.88 \mathrm{~ms}$, $S D=90.84 ; F(1,49)=10.94, p<.01)$. Reaction times for the affordance and neutral trials did not differ significantly from each other, $F(1$, $49)=.031, p>$.5. A significant effect of category was also found $F(1$, $49)=4.22, p<.05$, with responses to natural object targets $(M=552.76 \mathrm{~ms}, S D=95.63)$ being significantly faster than responses to manmade object targets $(M=601.76 \mathrm{~ms}, S D=77.08)$. There was no significant interaction between condition and target category, $F(3$, $147)=1.50, p>$.1.

\subsection{Electrophysiological results}

N200 analyses: comparison to baseline. The N200 was calculated by subtracting ERPs for go trials from those of nogo trials for the three priming conditions. For each condition, independent $t$-tests were used to compare the voltage of the N200 to a baseline of 0 across all temporal samples and active electrodes. These multiple comparisons were then corrected using the previously described cluster randomisation procedure. Significant clusters are listed in Table 1 and scalp maps illustrating the location of activity are shown in Fig. 2. This indicates that the information contingent on the semantic decision task (natural vs. manmade categorisation) was available from $208 \mathrm{~ms}$ after target word onset when primed with a semantically related word, from $252 \mathrm{~ms}$ when primed with a word sharing a similar micro-affordance and from $280 \mathrm{~ms}$ when it was primed with a neutral word.

N200 analyses: comparison between conditions. Independent $t$ tests were also carried out to examine the difference between the N200s of the different conditions. Again, multiple comparisons were corrected using the cluster randomisation procedure. The resulting significant clusters are shown in Table 2 and displayed in Fig. 3.

When subtracting the neutral from the semantic priming condition the presence of an early negative cluster (cS-N1) shows that the onset of the N200 was significantly earlier for semantically primed compared to neutrally primed target words. The later positive cluster (cS-N2) also

Table 1

Summary of significant clusters for the N200 of the semantic, affordance, and neutral conditions compared to baseline.

\begin{tabular}{llllll}
\hline Condition & $\begin{array}{l}\text { No. of } \\
\text { clusters }\end{array}$ & $\begin{array}{l}\text { Name of } \\
\text { cluster }\end{array}$ & Polarity & Duration & $P$-value \\
\hline Semantic & 1 & cS & Negative & $208-800 \mathrm{~ms}$ & $p<.001$ \\
Affordance & 1 & cA & Negative & $252-800 \mathrm{~ms}$ & $\begin{array}{c}p<.001 \\
\text { Neutral }\end{array}$ \\
1 & cN & Negative & $280-800 \mathrm{~ms}$ & $p<.001$ \\
\hline
\end{tabular}



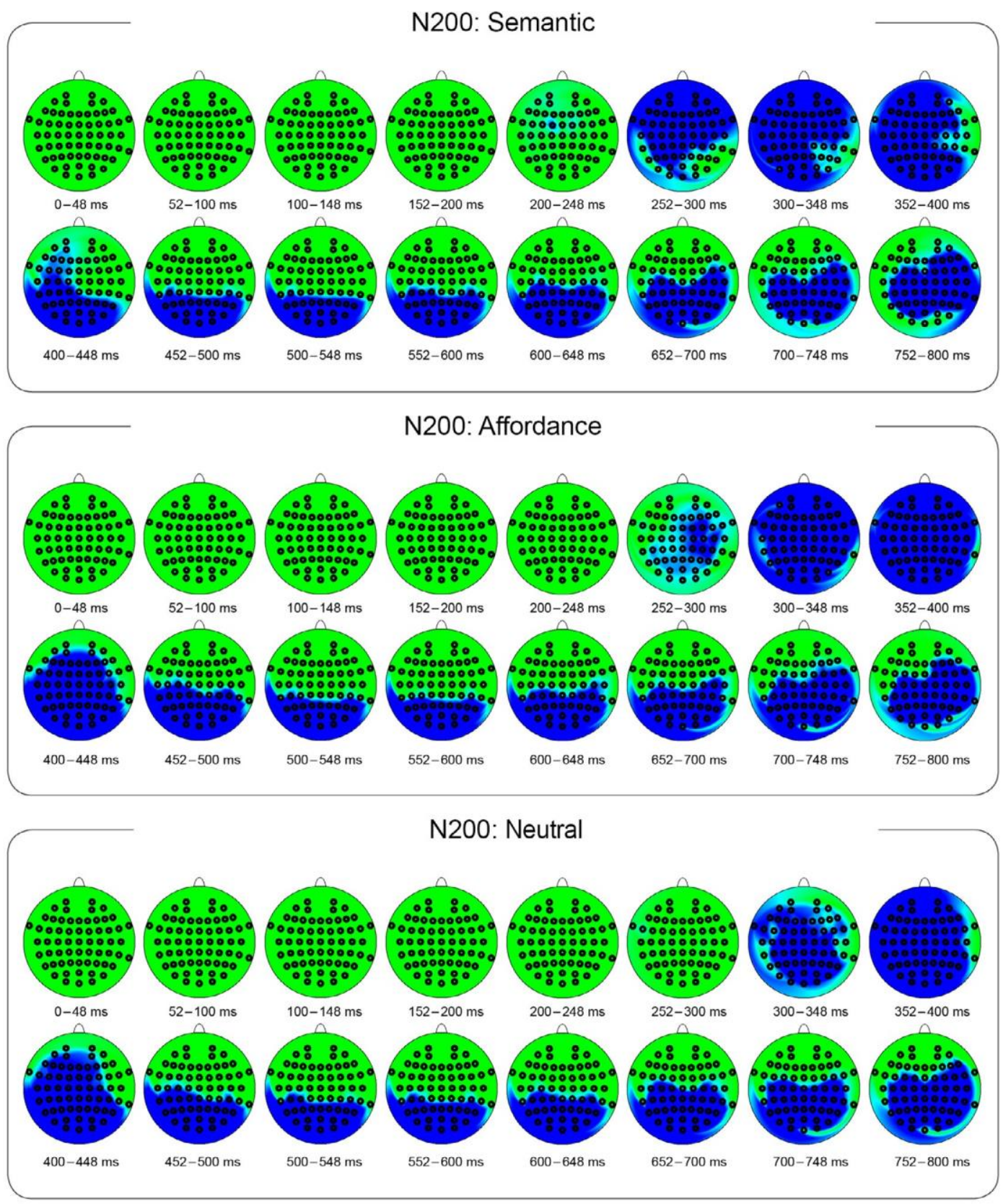

\section{t-score}

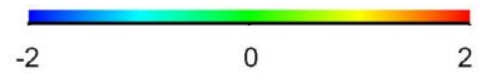

Fig. 2. Scalp maps showing $t$-scores for significant clusters revealed by the cluster randomisation comparison between the N200 and baseline for semantic, affordance, and neutral priming conditions.

Table 2

Summary of significant clusters in the comparisons between the N200 of the semantic, affordance, and neutral conditions.

\begin{tabular}{|c|c|c|c|c|c|}
\hline Conditions compared & No. of clusters & Name of cluster & Polarity & Duration & $P$-value \\
\hline \multirow{2}{*}{ Semantic - Neutral } & 2 & cS-N1 & Negative & $176-300 \mathrm{~ms}$ & $p=.002$ \\
\hline & & cS-N2 & Positive & $368-500 \mathrm{~ms}$ & $p<.001$ \\
\hline Affordance - Neutral & 1 & $\mathrm{cA}-\mathrm{N}$ & Negative & $200-292 \mathrm{~ms}$ & $p<.001$ \\
\hline Semantic - Affordance & 1 & cS-A & Positive & $320-500 \mathrm{~ms}$ & $p<.001$ \\
\hline
\end{tabular}



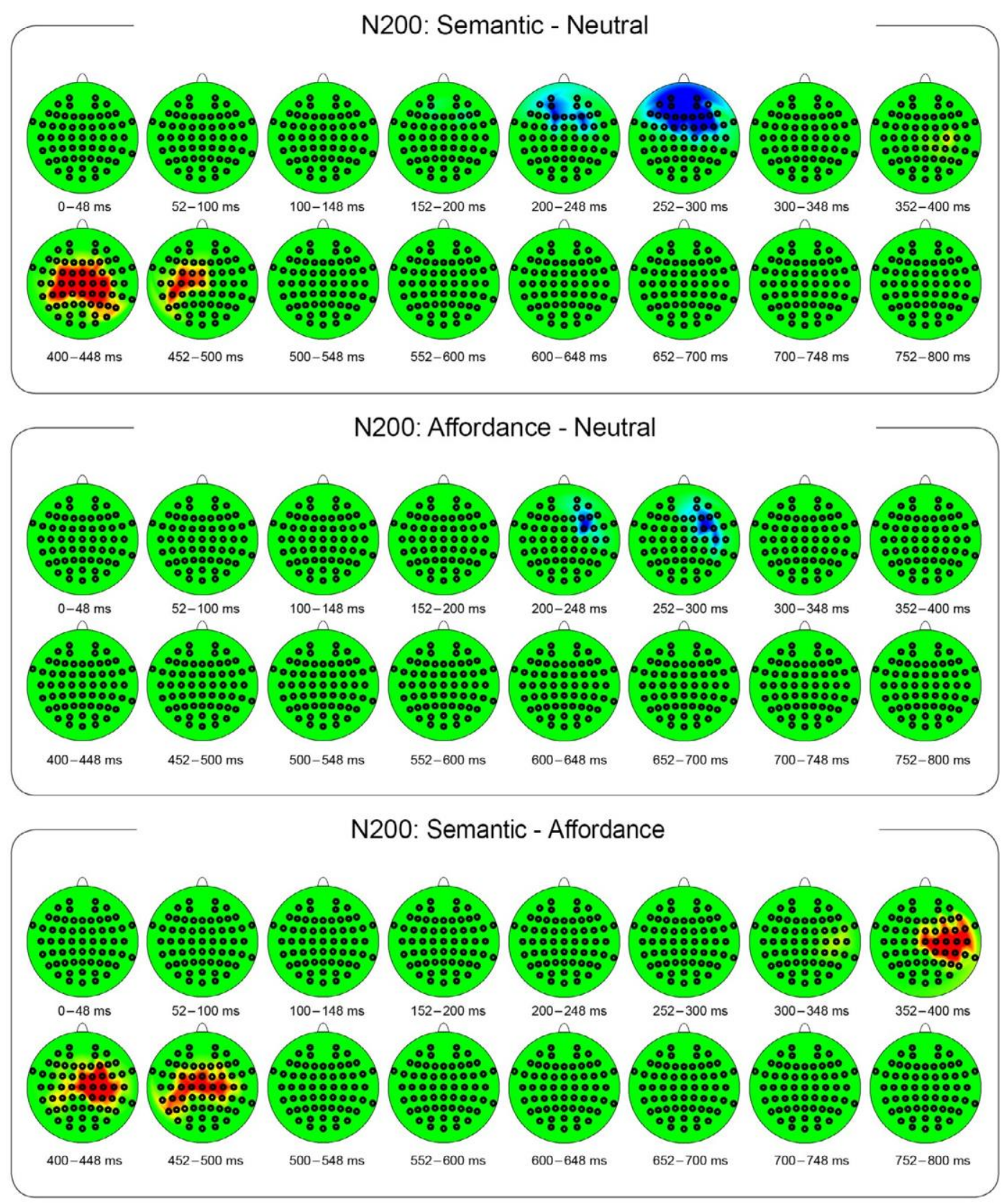

\section{t-score}

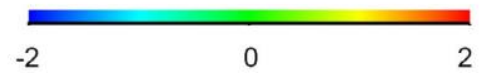

Fig. 3. Scalp maps showing $t$-scores for the significant clusters revealed by the cluster randomisation comparing semantic, affordance, and neutral priming N200s.

indicates that the offset of the N200 is earlier in the semantic than the neutral priming condition. Similar comparisons also revealed that the N200 was earlier in the affordance priming condition than the neutral priming condition (cluster cA-N). However, there was no significant difference between the latency of the onset of the N200 between the semantic priming condition and the affordance priming condition, only that the offset of the N200 was earlier in the former condition (cluster cS-A).

Summary of N200 results. Given that the onset of the N200 is thought to reveal the earliest time a participant has access to information required to make their task-related judgement, it is normal to find that onset latencies for this component are highly correlated to behavioural reaction times. This was indeed the case when comparing our semantic and neutral priming conditions, with the reaction times and N200 onset both being earlier in the semantic than the neutral priming condition. However, while reaction times were found to be significantly faster in the semantic priming condition than the affordance priming condition, we found no significant difference in the onset of the N200 between these conditions. A logical explanation for this disparity lies in the fact that reaction times are only garnered during go trials, while the N200 is the result of a subtraction of ERP between go and nogo trials. Thus, it is possible that the relatively early onset of the N200 for affordance priming is a result of greater activity during nogo trials, which would not be reflected in reaction times. Therefore, to test 
Table 3

Summary of significant clusters in the comparisons between the go trial ERPs of the semantic, affordance, and neutral conditions

\begin{tabular}{|c|c|c|c|c|c|}
\hline Condition & No. of clusters & Name of cluster & Polarity & Duration & $P$-value \\
\hline Semantic - Neutral & 1 & cS-N-Go & Negative & $428-708 \mathrm{~ms}$ & $\mathrm{p}<.001$ \\
\hline Affordance - Neutral & 1 & cA-N-Go & Negative & $520-728 \mathrm{~ms}$ & $p=.001$ \\
\hline Semantic - Affordance & 1 & cS-A-Go & Negative & $440-556 \mathrm{~ms}$ & $p<.001$ \\
\hline
\end{tabular}
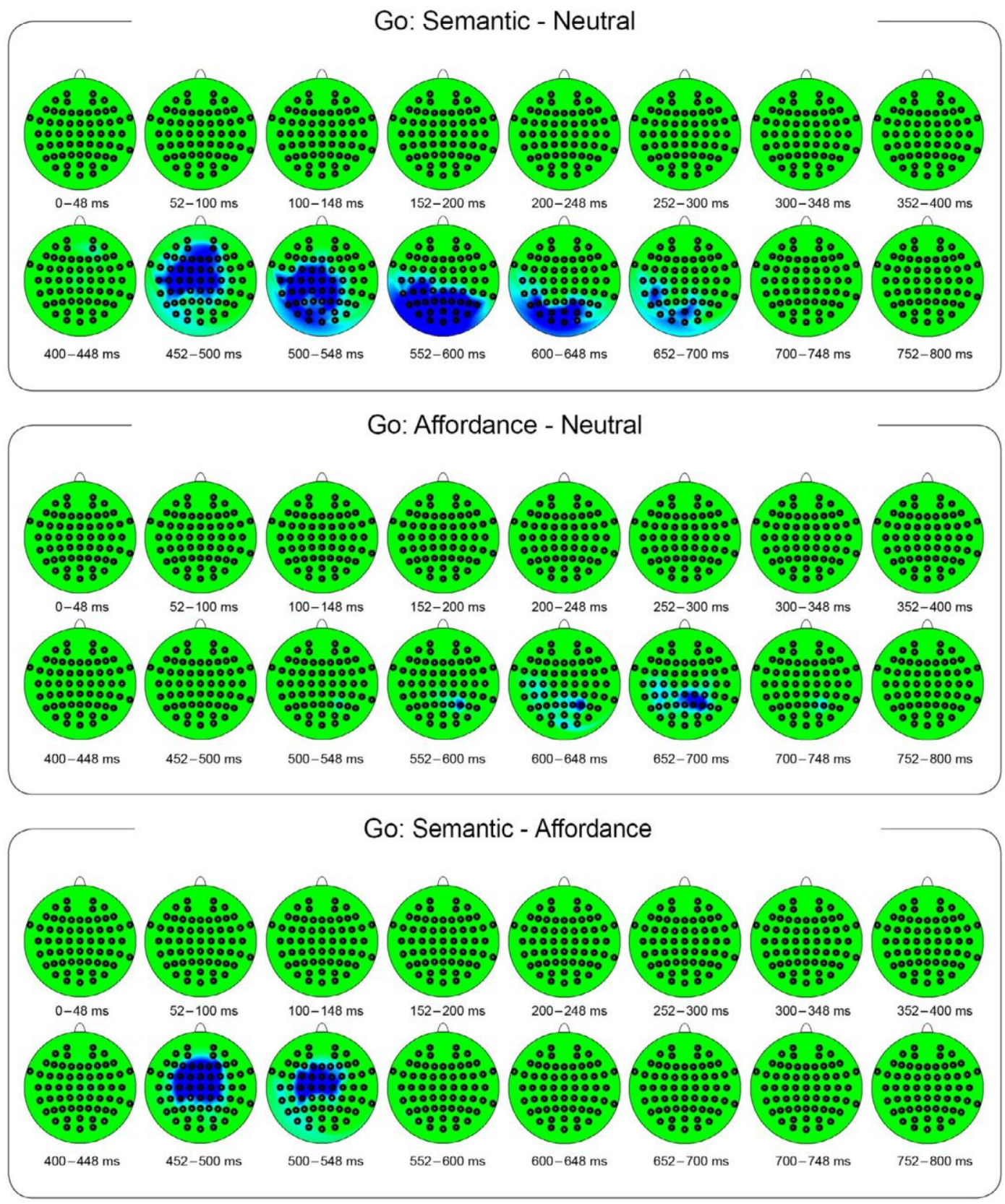

t-score

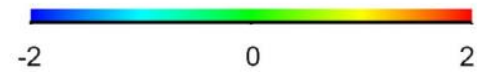

Fig. 4. Scalp maps showing $t$-scores for significant clusters revealed by the cluster randomisation comparison between semantic, affordance, and neutral priming conditions in go trials. 
Table 4

Summary of significant clusters in the comparisons between the nogo trial ERPs of the semantic, affordance, and neutral conditions.

\begin{tabular}{|c|c|c|c|c|c|}
\hline Condition & No. of clusters & Name of cluster & Polarity & Duration & $p$-value \\
\hline Semantic - Neutral & 1 & cS-N-Nogo & Negative & $500-632 \mathrm{~ms}$ & $p=.001$ \\
\hline Affordance - Neutral & 1 & cA-N-Nogo & Negative & $176-352 \mathrm{~ms}$ & $p=.001$ \\
\hline \multirow[t]{2}{*}{ Semantic - Affordance } & 2 & cS-A1-Nogo & Positive & $296-472 \mathrm{~ms}$ & $p=.002$ \\
\hline & & cS-A2-Nogo & Negative & $492-628 \mathrm{~ms}$ & $p<.001$ \\
\hline
\end{tabular}

this hypothesis we conducted separate analyses of ERP for go and nogo trials.

Analyses of go trials. Paired sample $t$-tests were used to compare the ERPs of the semantic and neutral priming conditions for go trials only. As before, individual $t$-tests were conducted for each sample recorded over each of the electrodes and multiple comparisons then corrected using the cluster randomisation procedure. The significant clusters are displayed in Table 3 and Fig. 4. Both semantic and affordance related priming evoked earlier activity in go-trial targets when compared with the neutral priming condition. However, activity for semantic primed targets occurred $\sim 100 \mathrm{~ms}$ earlier than those primed with affordance, as indicated by the presence of the negative polarity cluster (cS-A-Go).

Analyses of nogo trials. The significant clusters resulting from the cluster randomisation procedure for nogo trials are displayed in Table 4 and Fig. 5. As in the go trials, both semantic and affordance priming of targets in nogo trials resulted in significantly earlier activity than in the neutral priming condition. However, in this case, a direct comparison of semantic and affordance priming showed that the onset of activity was significantly earlier in the affordance priming condition, as revealed by the positive polarity cluster (cS-A1-Nogo). The later cluster (cS-A2Nogo) did reveal greater negative amplitudes due to semantic priming, but later, from 492 to $628 \mathrm{~ms}$.

\section{Discussion}

In this study, we examined the relative time course of access to an object's semantic or action-affordance features when reading its name. The availability of these different types of information was ascertained through an examination of the N200 ERP component, which can be used to determine when specific task-related information becomes available to a participant in a go/nogo paradigm. In our study participants made a semantic decision related to a written object name, categorising the described object as either manmade or natural. These target object names were primed by written words that were related to the target through a shared affordance, semantic taxonomy or were unrelated to the target in a neutral priming condition.

Our results showed that the onset of the N200 was earlier when the target words were primed with either semantic or affordance related primes, at around 210-250 ms post target onset, than when they were preceded with an unrelated word, where the onset was at $280 \mathrm{~ms}$. Importantly, there was no significant difference in N200 onset latency between affordance and semantic related primes. This would normally indicate that the two types of related priming conditions offer equivalent facilitation of the semantic decision made on the target words. However, this was found to be at odds with the behavioural reaction time data. Only semantic priming facilitated decision latencies, with latencies during the priming of affordances not differing significantly from the neutral priming condition. The explanation we pursued to explain for this disparity relates to the methodology used to calculate the N200 component, which is a difference wave of stimuli presented in go and nogo trials. This analysis draws upon all of the trials tested in the experiment, while reaction times are only provided for go trials. The disparity between reaction times and N200 latencies suggests that there is an asymmetric difference in ERPs between go and nogo trials that is modulated by the priming condition. From an embodied perspective this would not be unexpected, as it is possible that the motor preparation afforded by the objects described in the prime words could interact with a later task-related manual response. To investigate this explanation, we conducted separate ERP analyses for go and nogo trials. In go trials we found that the onset of activity in the semantic priming condition was significantly earlier than the other two priming conditions, starting around $430 \mathrm{~ms}$ after target onset. While the onset of activity in the affordance priming condition was earlier than that of the neutral condition, this difference was relatively late, at around $520 \mathrm{~ms}$. This could explain why affordance priming had no significant effect on reaction times, as the associated activity was proximal to the behavioural response times of around $560-580 \mathrm{~ms}$ and therefore too late to influence those responses. Thus, it would appear that the temporal aspect of go-response ERPs is in line with the behavioural latency differences. Conversely, in the ERPs from nogo trials the effect of affordance priming started at $180 \mathrm{~ms}$, significantly earlier than semantic priming at around $500 \mathrm{~ms}$. This comparison of go and nogo trials shows how the parity in the N200 onset in semantic and affordance priming conditions mask quite different underlying activities, with early activation of semantic representations in go trials, and early affordance related activity in nogo trials.

In part, these findings follow established semantic priming results, with activation from the prime word facilitating the activation of the proceeding semantically related targets. This is clearly evident in the semantic priming condition, where we find facilitation of behavioural semantic decision latencies, and early activity in the go trial ERPs. We also found activity related to affordance priming in go trials, but in a much later temporal window. Normally, these particular results would be indicative of a post-lexical mental simulation account of affordance (Mahon \& Caramazza, 2008) but they are at odds with other studies that have found an affordance-related facilitation in lexical decision latency (Myung et al., 2006; Rueschemeyer, Lindemann, van Rooij, van Dam, \& Bekkering, 2010).

One potential reason why we did not find a behavioural effect of affordance priming previously seen in lexical decisions is due to the task used in our study. This is not related particularly to the semantic decision task per se, but rather the relationship between the task and the two priming conditions. In the semantic priming condition, the taxonomic relation between prime and target ensured that they would share the semantic feature directly probed by the task i.e. prime and target would either both be natural or both be manmade. In contrast, the primes and targets in the affordance condition were selected such that their only shared feature should be the grip used to manipulate them. As such, natural primes would always be paired with artificial targets and vice versa. Thus, prime-target pairs in the affordance condition are always unrelated with respect to the attended semantic feature directed by the behavioural task (i.e. natural vs manmade). This means that the semantic priming condition has a direct relationship between prime, target, and task, whereas in the affordance condition the relationship between prime and target was orthogonal to the task. Therefore, it is possible that reaction times in affordance and semantic priming conditions may have been modulated by differences in the relationship between prime and task, rather than the relationship between prime and target. It must be noted, that this distinction cannot be applied to the temporal disparities shown in the study by Amsel et al. (2013), as they compared the temporal onset of distinct and explicit semantic 

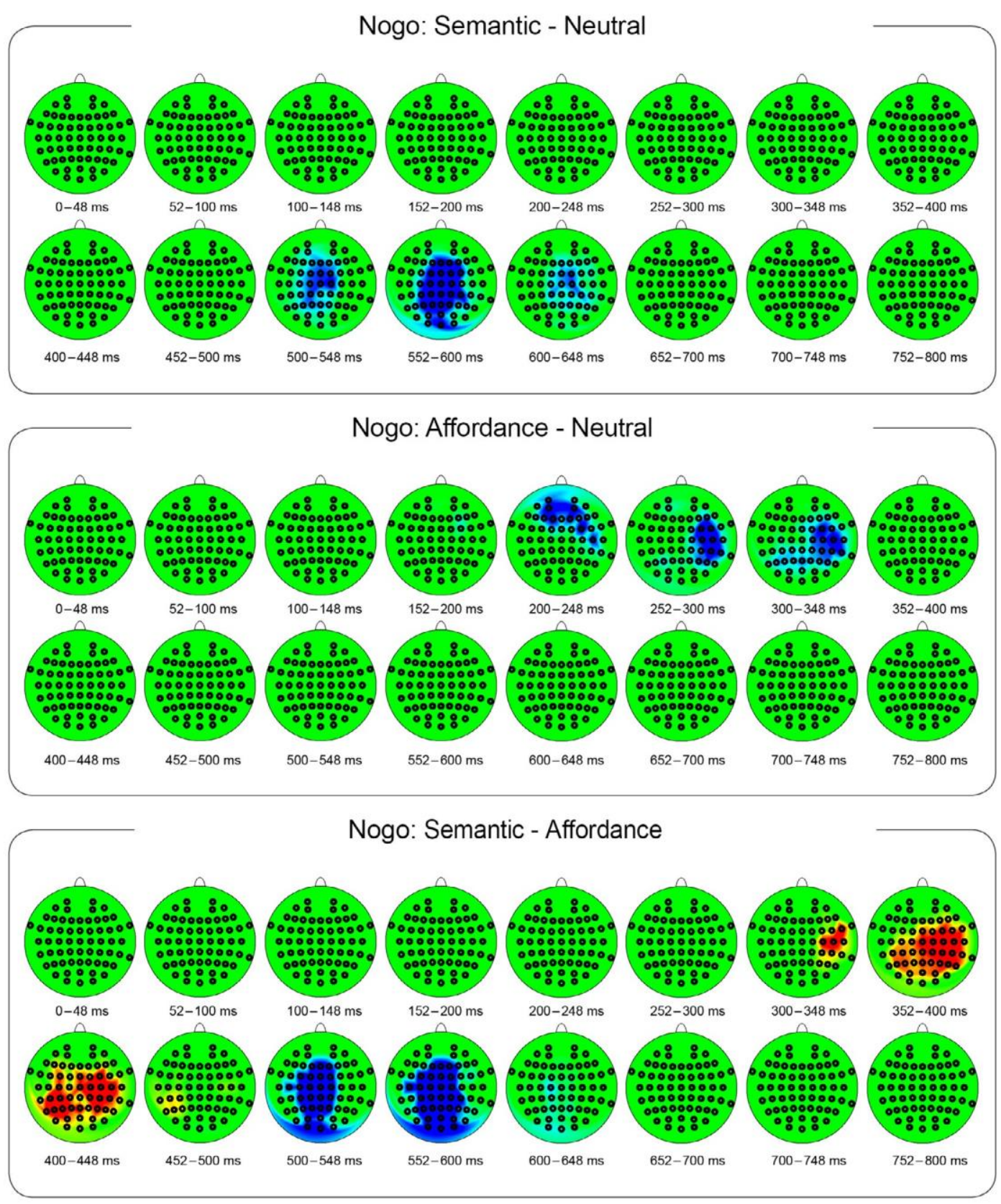

\section{t-score}

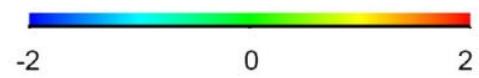

Fig. 5. Scalp maps showing $t$-scores for the significant clusters revealed by the cluster randomisation comparison between semantic, affordance, and neutral priming conditions in nogo trials.

decisions, rather than comparisons of priming in the same decision.

In Amsel et al. (2013) the onset of the N200 ERP component revealed that participants were able to differentiate between living and non-living entities from as early as $160 \mathrm{~ms}$, whereas the graspability of the object was retrieved around $300 \mathrm{~ms}$. It is concluded that this relatively late access to grasp-related affordances indicates that they do not play a crucial role in the conceptual representation of objects. In our own study, N200 latencies when priming affordance and semantic features of target words were equivalent, from around $210 \mathrm{~ms}$. Although similar, there are some important methodological divergences between our two studies that could explain the differences between our
N200 latencies. Firstly, the affordances related to Amsel et al.'s stimuli appeared to be based on the object's geometry, rather than stored representation of use. For example, a "mouse" (the small rodent not the hand-held device) is categorised as graspable, but a "motorbike" is not. In terms of affordances, the experience one has with using or manipulating an object is an important aspect of the sensorimotor activity elicited (Calvo-Merino, Glaser, Grèzes, Passingham, \& Haggard, 2005; Rueschemeyer et al., 2010; Siakaluk et al., 2008). Therefore, one could categorise a motorbike as a "graspable" object, as it is manipulated primarily through its handlebars. Conversely, a mouse is unlikely to have a stored affordance, generated through past interactions, but 
would rely upon an intrinsic geometric affordance inferred from its size. Physical size provides a salient affordance in visual stimuli, with direct vision-to-action activation obviating the requirement for higher level knowledge (Rumiati \& Humphreys, 1998). However, in a linguistic modality, geometric affordances have to be mediated through stereotyping of object properties. Therefore, in Amsel et al. the temporal onset of affordance seen in the N200 would have included any delay required to infer a geometric affordance. In contrast, our study used a combination of stored and geometric affordance for each prime/target pair. This could be particularly important given that Amsel et al. based their temporal estimates upon a manipulation of the question posed in an explicit decision task, i.e. between making a living/non-living or graspable/non-graspable judgment.

One of our central questions is whether the availability of implied geometric information to an explicit decision task, as tested in Amsel et al., can provide an ecologically valid estimate of the temporal onset of affordance. We suggest that this is challenged by our finding of early activity associated with affordance priming in nogo trials, a con-sequence of the manual motor preparation generated by the shared affordances between prime and target. In nogo trials this motor pre-paration is incompatible with the requirement of the participant to withhold a manual response, and the inhibition of the response be-comes evident in the ERP. The spatial distribution of this activity, evident in right prefrontal electrodes, is similar to the activity found in previous research in the right inferior frontal gyrus during the inhibi-tion of motor responses (Chikazoe, Konishi, Asari, Jimura, \& Miyashita, 2007; Hampshire, Chamberlain, Monti, Duncan, \& Owen, 2010). Lesion studies also illustrate the involvement of the frontal lobe in inhibiting affordances that are automatically elicited by visual objects. One study found that some patients with frontal lobe lesions would grasp and use any objects in their field of vision without any real purpose for doing so (Lhermitte, 1983; Riddoch, Edwards, Humphreys, West, \& Heafield, 1998). Lhermitte (1983) argued that this 'utilization behaviour', as he termed it, resulted from the inability of the frontal lobe to perform the usual inhibitory function on the parietal lobe's motor programs. Re-search shows how the parietal lobe is involved in the integration of visual and somatosensory information and converting this into motor commands (see Fogassi \& Luppino, 2005, for a review). The temporal period of this affordance related activity, spanning $\mathrm{P} 2$ and N2 compo-nents, is also consistent with previous accounts of these components reflecting stimulus evaluation and response selection (Gajewski, Stoerig, \& Falkenstein, 2008; Potts, 2004). Gajewski et al. (2008) ar-gued that this might be more effortful when an incorrect response is activated by misleading cues. We posit that there is a similar modula-tion of the P2/N2 complex in our study due to the effort required to inhibit a response once the motor system had been primed to respond. We suggest that this is the source of the early activity observed in our affordance primed nogo trials.

A particularly noteworthy aspect of this account of our affordance effects is that the task-related manual response was not directly related to the affordance of the object. While participants were asked to re-spond or withhold a manual response, the left index finger button press required was not directly related to the grip afforded by either the prime or target referent object. Similarly, generalised effects have been found in previous research (Postle, Ashton, McFarland, \& De Zubicaray, 2013; Rueschemeyer et al., 2010; Siakaluk et al., 2008). Rueschemeyer et al. found that lexical decisions were quicker and more accurate for names of manipulable objects when participants simultaneously exe-cuted a motor action. In this case the action, requiring participants to run their finger along the edge of a desk, was not specific to the af-forded actions of the objects. Postle et al. also found that it didn't matter which body part was being described by their linguistic stimuli, the right hand was affected indifferently. This shows that the motor preparation generated through affordance priming does not necessarily have to be related to a specific motor program, such as a particular grip, but can be broadly tuned to include other manual activity.

\section{Conclusions}

The main aim of this study was to investigate the deeply embodied claim that sensorimotor information related to the form or function of an object, is fundamental to its conceptual representation and plays a privileged role in the comprehension of their linguistic descriptors (Glenberg \& Gallese, 2012; Glenberg \& Robertson, 1999). Conversely, it has been suggested that access to action-affordances is not privileged above other semantic features that make up an object's conceptual representation, and that their activation is a result of post-lexical mental simulation of referent object use (e.g. Amsel et al., 2013; Mahon \& Caramazza, 2008). In support of the former theory, we established that the priming of affordance evokes the rapid activation of motor representation during the reading of object names. This is indicative of somatotopic activity in the motor system associated with the affordance of the named object, similar to that shown across a range of studies during the reading of action words (Hauk, Shtyrov, \& Pulvermüller, 2008; Pulvermüller, Härle, \& Hummel, 2001; Pulvermüller, Lutzenberger, \& Preissl, 1999; Shtyrov, Hauk, \& Pulvermüller, 2004). However, while listeners do seem able to extract embodied information from linguistic representations, this information does not appear to play a fundamental role in the semantic integration processes related to our task. The early activity related to affordance priming, seen from around $180 \mathrm{~ms}$, was strictly limited to nogo trials. This indicates that this activity is related to the inhibition of the afforded motor preparation, as participants seek to withhold the manual response related to the task. While taxonomically related primes facilitated semantic decisions, affordance related primes did not. In go trials the onset of activity related to affordance priming was relatively late, starting at around $520 \mathrm{~ms}$, compared to an onset of $430 \mathrm{~ms}$ for semantic priming. This set of findings is perhaps most consistent with theories that posit multiple processing routes in comprehension, such as the language and situated simulation (LASS) theory (Barsalou et al., 2008). This would allow for a distinction between the early activation of the motor representation afforded by the described object and the route used to access abstract conceptual information during comprehension. Whereas semanticpriming can occur by recruiting the linguistic system's method of activating associated words in a distributional semantic network, affordance-priming involves the situated simulation route. Our research suggests that the early motor simulation activity is activated automatically and in a similar time frame to categorical knowledge. Later affordance related activation could either be the result of another simulation cycle or integration of information across the two routes.

\section{Statement of significance}

From examining the event-related potentials of participants reading object names, we found early activation of object affordances from $176 \mathrm{~ms}$. This suggests affordances are activated during an earlier stage of linguistic processing than previously thought. In the article, we explain why current research methodologies may have failed to find similar results.

\section{Acknowledgements}

We would like to thank Steven Feven-Parsons for creating the graphics for this paper. 


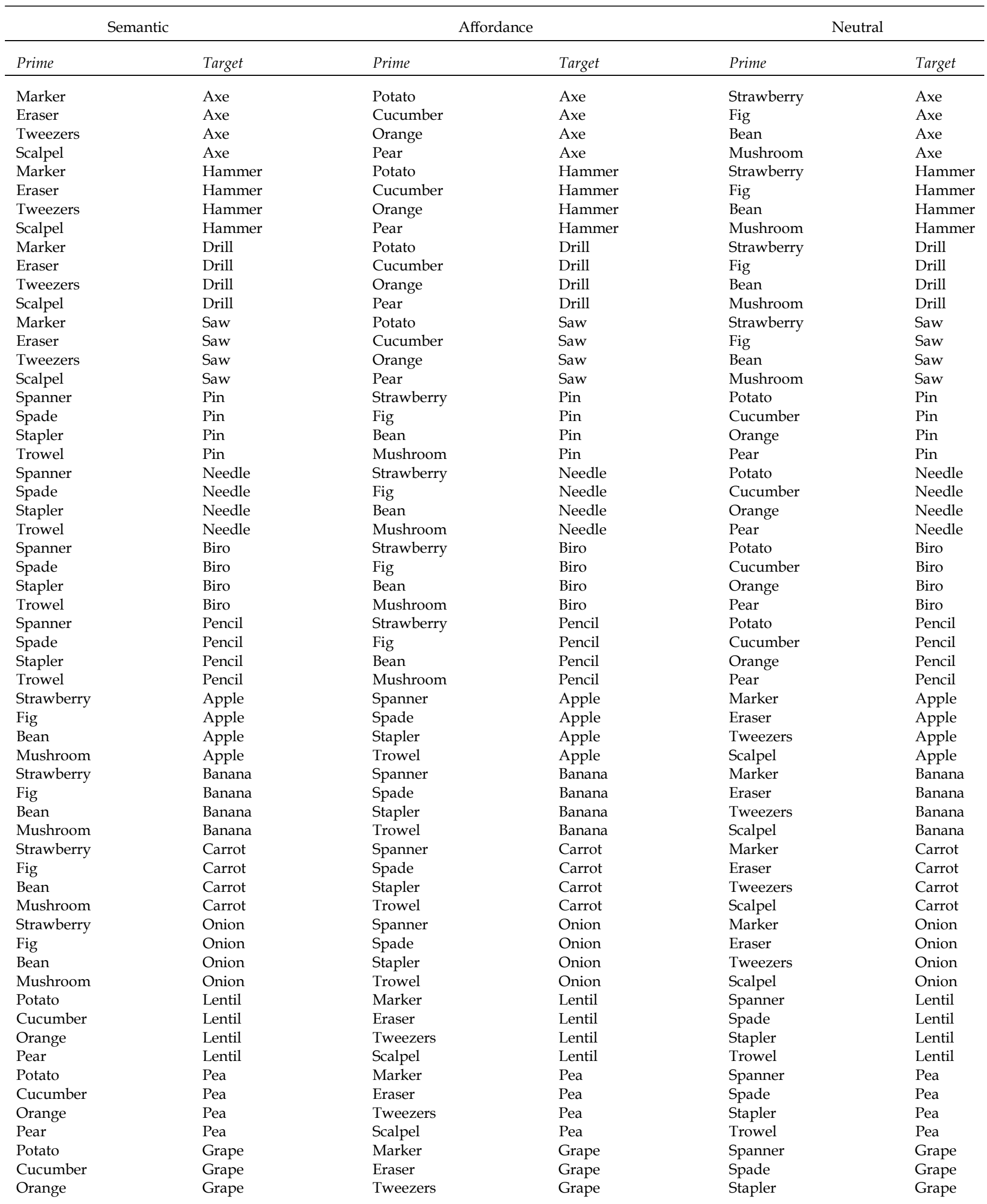




\begin{tabular}{|c|c|c|c|c|c|}
\hline Pear & Grape & Scalpel & Grape & Trowel & Grape \\
\hline Potato & Cherry & Marker & Cherry & Spanner & Cherry \\
\hline Cucumber & Cherry & Eraser & Cherry & Spade & Cherry \\
\hline Orange & Cherry & Tweezers & Cherry & Stapler & Cherry \\
\hline Pear & Cherry & Scalpel & Cherry & Trowel & Cherry \\
\hline
\end{tabular}

\section{References}

Amsel, B. D., Urbach, T. P., \& Kutas, M. (2013). Alive and grasping: Stable and rapid semantic access to an object category but not object graspability. Neuroimage, 77, 113.

Augustin, M. D., Defranceschi, B., Fuchs, H. K., Carbon, C. C., \& Hutzler, F. (2011). The neural time course of art perception: An ERP study on the processing of style versus content in art. Neuropsychologia, 49(7), 2071-2081.

Baayan, H., Piepenbrock, R., \& Gulikers, L. (1995). The CELEX lexical database (CDROM). University of Pennsylvania, Philadelphia: Linguistic Data Consortium.

Barbieri, F., Buonocore, A., Bernardis, P., Dalla Volta, R., \& Gentilucci, M. (2007). On the relations between affordance and representation of the agent's effector. Experimental Brain Research, 180(3), 421-433.

Barsalou, L. W., Santos, A., Simmons, W. K., \& Wilson, C. D. (2008). Language and simulation in conceptual processing. In M. De Vega, A. M. Glenberg, \& A. C. Graesser (Eds.). Symbols, embodiment, and meaning (pp. 245-283). Oxford: Oxford University Press.

Battig, W. F., \& Montague, W. E. (1969). Category norms for verbal items in 56 categories: A replication and extension of the Connecticut norms. Journal of Experimental Psychology, 80, 1-46.

Borovsky, A., Elman, J. L., \& Kutas, M. (2012). Once is enough: N400 indexes semantic integration of novel word meanings from a single exposure in context. Language Learning and Development, 8(3), 278-302.

Bub, D. N., \& Masson, M. E. (2012). On the dynamics of action representations evoked by names of manipulable objects. Journal of Experimental Psychology, 141(3), 502-517.

Bub, D. N., Masson, M. E., \& Cree, G. S. (2008). Evocation of functional and volumetric gestural knowledge by objects and words. Cognition, 106(1), 27-58.

Calvo-Merino, B., Glaser, D. E., Grèzes, J., Passingham, R. E., \& Haggard, P. (2005). Action observation and acquired motor skills: An FMRI study with expert dancers. Cerebral Cortex, 15(8), 1243-1249.

Chatterjee, A. (2010). Disembodying cognition. Language and Cognition, 2(1), 79-116.

Chikazoe, J., Konishi, S., Asari, T., Jimura, K., \& Miyashita, Y. (2007). Activation of right inferior frontal gyrus during response inhibition across response modalities. Journal of Cognitive Neuroscience, 19(1),69-80.

Deacon, D., Hewitt, S., Yang, C. M., \& Nagata, M. (2000). Event-related potential indices of semantic priming using masked and unmasked words: Evidence that the N400 does not reflect a post-lexical process. Cognitive Brain Research, 9(2), 137-146.

Dove, G. (2009). Beyond perceptual symbols: A call for representational pluralism. Cognition, 110(3), 412-431.

Dove, G. (2011). On the need for embodied and dis-embodied cognition. Embodied and Grounded Cognition, 129.

Fogassi, L., \& Luppino, G. (2005). Motor functions of the parietal lobe. Current Opinion in Neurobiology, 15(6), 626-631.

Forster, K. I., \& Davis, C. (1984). Repetition priming and frequency attenuation in lexical access. Journal of Experimental Psychology: Learning, Memory, and Cognition, 10(4), 680698.

Gajewski, P. D., Stoerig, P., \& Falkenstein, M. (2008). ERP-correlates of response selection in a response conflict paradigm. Brain Research, 1189, 127-134.

Garbarini, F., \& Adenzato, M. (2004). At the root of embodied cognition: Cognitive science meets neurophysiology. Brain and Cognition, 56(1), 100-106.

Gentilucci, M., \& Gangitano, M. (1998). Influence of automatic word reading on motor control. European Journal of Neuroscience, 10(2), 752-756.

Gibson, J. (1979). The theory of affordances in the ecological approach to visual perceptual. Houghton Mifflin.

Glenberg, A. M., \& Gallese, V. (2012). Action-based language: A theory of language acquisition, comprehension, and production. Cortex, 48(7), 905-922.

Glenberg, A. M., \& Robertson, D. A. (1999). Indexical understanding of instructions. Discourse Processes, 28(1), 1-26.

Glover, S., \& Dixon, P. (2002). Semantics affect the planning but not control of grasping. Experimental Brain Research, 146(3), 383-387.

Glover, S., Rosenbaum, D. A., Graham, J., \& Dixon, P. (2004). Grasping the meaning of words. Experimental Brain Research, 154(1), 103-108.

Grabowski, T. J., Damasio, H., \& Damasio, A. R. (1998). Premotor and prefrontal correlates of category-related lexical retrieval. Neuroimage, 7(3), 232-243.

Hampshire, A., Chamberlain, S. R., Monti, M. M., Duncan, J., \& Owen, A. M. (2010). The role of the right inferior frontal gyrus: Inhibition and attentional control. Neuroimage, 50(3), 1313-1319.

Hauk, O., Shtyrov, Y., \& Pulvermüller, F. (2008). The time course of action and actionword comprehension in the human brain as revealed by neurophysiology. Journal of Physiology-Paris, 102(1), 50-58.

Kutas, M., \& Federmeier, K. D. (2011). Thirty years and counting: Finding meaning in the N400 component of the event-related brain potential (ERP). Annual Review of Psychology, 62, 621-647.

Lhermitte, F. (1983). 'Utilization behaviour'and its relation to lesions of the frontal lobes. Brain, 106(2), 237-255.

Lucas, M. (2000). Semantic priming without association: A meta-analytic review. Psychonomic Bulletin \& Review, 7(4), 618-630.

Mahon, B. Z., \& Caramazza, A. (2008). A critical look at the embodied cognition hypothesis and a new proposal for grounding conceptual content. Journal of Physiology Paris, 102(1), 59-70.

Marino, B. F., Gough, P. M., Gallese, V., Riggio, L., \& Buccino, G. (2013). How the motor system handles nouns: A behavioral study. Psychological Research, 77(1), 64-73.

Maris, E., \& Oostenveld, R. (2007). Nonparametric statistical testing of EEG-and MEGdata. Journal of Neuroscience Methods, 164(1), 177-190.

Meyer, D. E., \& Schvaneveldt, R. W. (1971). Facilitation in recognizing pairs of words: Evidence of a dependence between retrieval operations. Journal of Experimental Psychology, 90(2), 227.

Myung, J.-Y., Blumstein, S. E., \& Sedivy, J. C. (2006). Playing on the typewriter, typing on the piano: Manipulation knowledge of objects. Cognition, 98(3), 223-243.

Oldfield, R. C. (1971). The assessment and analysis of handedness: The Edinburgh inventory. Neuropsychologia, 9(1), 97-113.

Postle, N., Ashton, R., McFarland, K., \& De Zubicaray, G. I. (2013). No specific role for the manual motor system in processing the meanings of words related to the hand. Frontiers in Human Neuroscience, 7.

Potts, G. F. (2004). An ERP index of task relevance evaluation of visual stimuli. Brain and Cognition, 56(1), 5-13.

Pulvermüller, F., Härle, M., \& Hummel, F. (2001). Walking or talking?: Behavioral and neurophysiological correlates of action verb processing. Brain and Language, 78(2), 143-168.

Pulvermüller, F., Lutzenberger, W., \& Preissl, H. (1999). Nouns and verbs in the intact brain: Evidence from event-related potentials and high-frequency cortical responses. Cerebral Cortex, 9(5), 497-506.

Pulvermüller, F., Shtyrov, Y., \& Ilmoniemi, R. (2005). Brain signatures of meaning access in action word recognition. Journal of Cognitive Neuroscience, 17(6), 884-892.

Riddoch, M. J., Edwards, M. G., Humphreys, G. W., West, R., \& Heafield, T. (1998). Visual affordances direct action: Neuropsychological evidence from manual interference. Cognitive Neuropsychology, 15(6-8), 645-683.

Rueschemeyer, S.-A., Lindemann, O., van Rooij, D., van Dam, W., \& Bekkering, H. (2010). Effects of intentional motor actions on embodied language processing. Experimental Psychology (formerly Zeitschrift für Experimentelle Psychologie), 57(4), 260-266.

Rumiati, R. I., \& Humphreys, G. W. (1998). Recognition by action: Dissociating visual and semantic routes to action in normal observers. Journal of Experimental Psychology: Human Perception and Performance, 24(2), 631.

Schmitt, B. M., Münte, T. F., \& Kutas, M. (2000). Electrophysiological estimates of the time course of semantic and phonological encoding during implicit picture naming. Psychophysiology, 37(4), 473-484.

Schneider, E., \& Zuccoloto, A. (2007). E-prime 2.0 [Computer software]. Pittsburg, PA: Psychological Software Tools.

Shtyrov, Y., Hauk, O., \& Pulvermüller, F. (2004). Distributed neuronal networks for encoding category-specific semantic information: The mismatch negativity to action words. European Journal of Neuroscience, 19(4), 1083-1092.

Siakaluk, P. D., Pexman, P. M., Sears, C. R., Wilson, K., Locheed, K., \& Owen, W. J. (2008). The benefits of sensorimotor knowledge: Body-object interaction facilitates semantic processing. Cognitive Science, 32(3), 591-605.

Tucker, M., \& Ellis, R. (2004). Action priming by briefly presented objects. Acta Psychologica, 116(2), 185-203.

Withagen, R., de Poel, H. J., Araújo, D., \& Pepping, G.-J. (2012). Affordances can invite behavior: Reconsidering the relationship between affordances and agency. New Ideas in Psychology, 30(2), 250-258. 